\title{
Detailed analysis of allergic cutaneous reactions to spinal cord stimulator devices
}

This article was published in the following Dove Press journal:

Journal of Pain Research

31 July 2013

Number of times this article has been viewed

\author{
Zeshan Ahmed Chaudhry' \\ Umer Najib ${ }^{2}$ \\ Zahid $\mathrm{H} \mathrm{Bajwa}^{3}$ \\ W Carl Jacobs ${ }^{4}$ \\ Javed Sheikh ${ }^{5}$ \\ Thomas T Simopoulos ${ }^{6}$ \\ 'Department of Diagnostic and \\ Interventional Neuroradiology, \\ Massachusetts General Hospital/ \\ Harvard Medical School, Boston, \\ MA, USA; ${ }^{2}$ Department of Neurology, \\ Robert C Byrd Health Sciences \\ Center of West Virginia University, \\ Morgantown, WV, USA; ${ }^{3}$ Boston \\ Headache Institute, Waltham, MA, \\ USA; ${ }^{4}$ Department of Pathology, Beth \\ Israel Deaconess Medical Center, \\ Harvard Medical School, Boston, \\ MA, USA; ${ }^{5}$ Department of Medicine, \\ Allergy and Immunology, ${ }^{6}$ Department \\ of Anesthesia, Beth Israel Deaconess \\ Medical Center, Brookline, MA, USA
}

Correspondence: Thomas T Simopoulos Department of Anesthesia, Beth Israel Deaconess Medical Center, Harvard Medical School, 330 Brookline Ave Boston, MA 02215, USA

Tel + I 617278803 I

Fax + I 6172788065

Email tsimopou@bidmc.harvard.edu

\begin{abstract}
The use of spinal cord stimulation (SCS) devices to treat chronic, refractory neuropathic pain continues to expand in application. While device-related complications have been well described, inflammatory reactions to the components of these devices remain underreported. In contrast, hypersensitivity reactions associated with other implanted therapies, such as endovascular and cardiac rhythm devices, have been detailed. The purpose of this case series is to describe the clinical presentation and course of inflammatory reactions as well as the histology of these reactions. All patients required removal of the entire device after developing inflammatory reactions over a time course of 1-3 months. Two patients developed a foreign body reaction in the lead insertion wound as well as at the implantable pulse generator site, with histology positive for giant cells. One patient developed an inflammatory dermatitis on the flank and abdomen that resolved with topical hydrocortisone. "In vivo" testing with a lead extension fragment placed in the buttock resulted in a negative reaction followed by successful reimplantation of an SCS device. Inflammatory reactions to SCS devices can manifest as contact dermatitis, granuloma formation, or foreign body reactions with giant cell formation. Tissue diagnosis is essential, and is helpful to differentiate an inflammatory reaction from infection. The role of skin patch testing for 96 hours may not be suited to detect inflammatory giant cell reactions that manifest several weeks post implantation.
\end{abstract}

Keywords: spinal cord stimulation, delayed inflammatory responses, foreign body giant cell reactions, contact dermatitis

\section{Introduction}

Spinal cord stimulation (SCS) has been used since the 1960s to treat chronic refractory pain conditions. In particular, SCS has been extensively used to manage the painful symptoms related to chronic lumbosacral radiculopathy following failed back surgery syndrome and complex regional pain syndromes. Randomized controlled trials of SCS for failed back surgery syndrome have reported favorable long-term results. ${ }^{1,2}$ Concurrently, there has been significant technologic advancement in the equipment for SCS. Specifically, implanting physicians can offer patients rechargeable implantable pulse generators, improved anchoring systems, and more stimulating lead contacts to ensure coverage for paresthesia in painful somatic regions. Thus, there is more widespread use of SCS therapy and acceptance among treating physicians.

Despite the positive findings and increasing experience of implanting physicians using SCS therapy, complications remain a common occurrence. An analysis of the available literature by Turner et al found that just over one third of patients have a complication. ${ }^{3}$ Prospective data from a multicenter evaluation of SCS for failed back 
surgery syndrome showed a similar rate of complications, even in the hands of experienced implanters. ${ }^{4}$ These adverse events are usually lead migration, infection, lead breakage, and unwanted stimulation. ${ }^{4,5}$ An allergic or immunologic/ inflammatory reaction to the system components is thought to be rare, and has been estimated by Cameron to be $0.1 \%{ }^{5}$ However, the true incidence may be underestimated because clinicians may fail to include it in the differential diagnosis, attributing an inflammatory reaction of the soft tissue surrounding SCS components to infection. In addition, only two articles detail the cutaneous reaction to the components of SCS systems. ${ }^{6,7}$ The purpose of this paper is to detail the presentation, clinical course, and histologic findings in three patients with cutaneous reactions to spinal cord stimulator equipment components.

\section{Case I}

The patient was a 61-year-old male who, despite two previous laminectomies, epidural injections, and multimodal analgesia (oxycodone/acetaminophen, cyclobenzaprine, ibuprofen), complained of severe pain secondary to left-sided chronic lumbosacral radiculopathy. The pain was 9/10 in intensity, located at his lower back on the left side with radiation down his posterior thigh, behind his knee to his lateral leg, and to the bottom of the sole of his foot. He had a past medical history of irritable bowel syndrome, squamous cell carcinoma of the skin, gastroesophageal reflux disease, and depression. The only reported allergy was to meperidine. Magnetic resonance imaging revealed no evidence of spinal stenosis, foraminal narrowing, or nerve root displacement, so he was implanted with an SCS consisting of two eight-contact leads, two lead extensions, and a rechargeable implantable pulse generator (EONTM, St Jude Medical Neuromodulation Division, Plano, TX, USA) following a successful trial of 7 days.

He was seen one week after the operation and his wounds, which included the abdomen for the generator, flank for the extension, and back for lead insertion and anchoring, were noted to be dry with intact incisions, and no erythema, drainage, or tenderness. He was afebrile. At one month post operation, the patient developed a left-sided rash on his flank and abdomen, with no fevers or chills (Figure 1). The stimulator was working well, providing up to $60 \%$ pain relief. The incisions over his abdomen, flank, and back were well healed. However, he had an area of erythema/dermatitis that seemed to be localized to the area over the length of the lead extensions connecting the implantable pulse generator to the leads. The reaction did not involve the implantable pulse generator site. It was noted that this rash appeared to

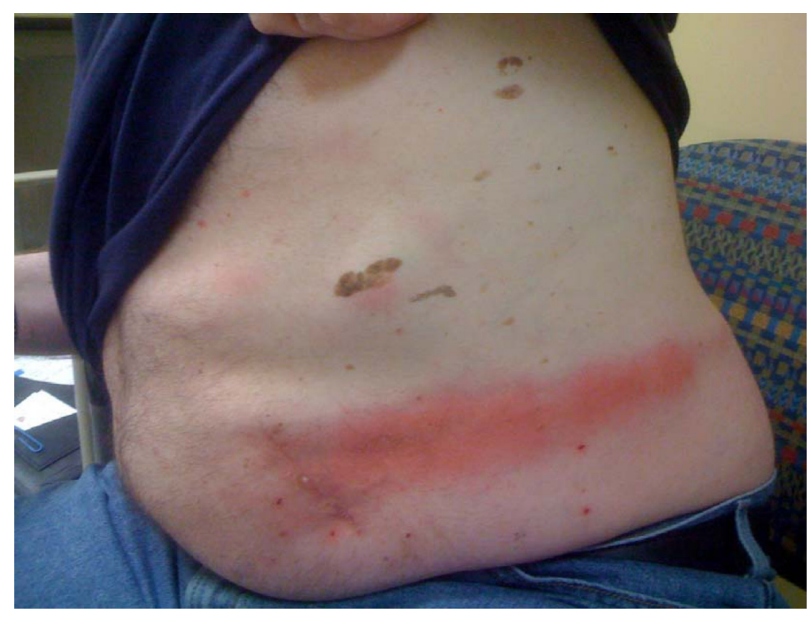

Figure I Erythematous rash on the abdomen and flank (case I) primarily overlying the lead extensions.

be an area of reactive inflammation, as opposed to infection, and he was diagnosed with a hypersensitivity reaction to the implanted wires. He was given topical $1 \%$ hydrocortisone cream and seen 7 days later, with resolution of the rash.

Forty-three days after implantation, the patient presented with complaints concerning wound drainage. There was no history of fall or trauma. The abdominal and flank incisions had dehisced, with device exposure and were draining pink, clear fluid. He was afebrile, with only mild tenderness of the wounds. His white blood cell count was 6.8 and the differential was abnormal for eosinophils at 5.4\%, with no bandemia or elevation in fraction of neutrophils. Because of concerns for potential early infection, urgent explantation of the entire SCS device was performed. Exploration of the wounds did not reveal any purulent material, but the subcutaneous tissue directly involving the wound was frail, bled easily with manipulation, and was difficult to coagulate. A tissue biopsy was taken from the lead extension site (Figure 2). Once cultures were sent, the patient was started on empiric vancomycin and ceftazidime.

Wound cultures were positive for rare coagulase negative Staphylococci as well as rare Staphylococcus aureus, and the patient was treated for 2 weeks with intravenous vancomycin. Clinically, however, he did not manifest with the usual clinical findings suggestive of device infection. One month later, all incisions were well healed. Because the initial reaction to the SCS equipment involved the flank to the abdomen but not the back, the buttock was targeted as a potential site for the implantable pulse generator. In an attempt to rule out a hypersensitivity reaction, an in vivo allergy test using a portion of the extension lead was done (Figure 3). A $4 \mathrm{~cm}$ lead extension piece was placed in the left buttock via a 12 gauge 


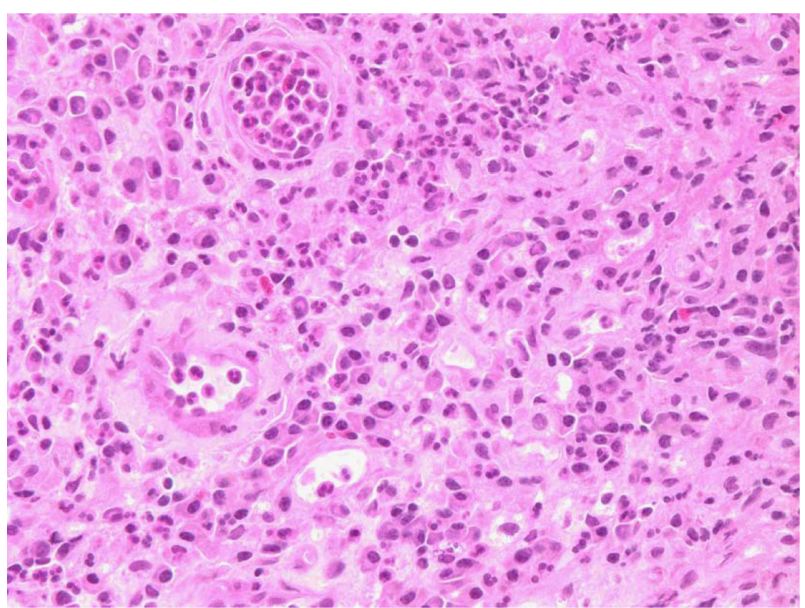

Figure 2 Histopathology of soft tissue removed from the lead extension wound at the flank (case I). Light microscopy revealed granulation tissue, necrotic debris, and fibrin with acute and chronic inflammation. No giant cell reaction was seen.

angiocatheter upon removal of the introducer. The extension fragment was placed into the catheter which was removed leaving the lead in the subcutaneous tissue. The puncture site was closed with 4.0 Monocryl ${ }^{\mathrm{TM}}$ suture (Ethicon Inc, Somerville, NJ, USA). After a 2-month follow-up period, there was no cutaneous reaction to the lead extension.

One month later, the system was reimplanted, with the pulse generator pocket site now in the left buttock. The patient tolerated the procedure well and had no postoperative complications. Now three years following reimplantation, his pain is well managed with a combination of SCS and medications.

\section{Case 2}

The patient was a 44-year-old gentleman with a past surgical history of decompression laminotomy, discectomy, and

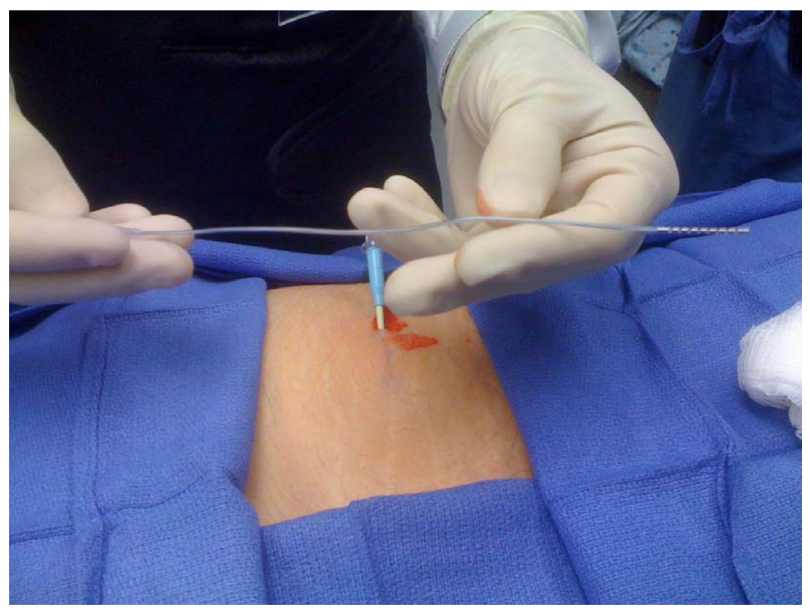

Figure 3 The distal $4 \mathrm{~cm}$ of the lead extension pictured above was inserted via a I 2 gauge angiocatheter into the subcutaneous tissue of the left buttock in case I for in vivo testing. fusion at L5/S1. One year following the surgery, the patient continued to have worsening low back pain, with radiation down his lateral calf into the lateral aspect of his dorsal foot. The patient described the pain as being $8 / 10$ in severity, with an achy quality that was partially alleviated with oxycodone/ acetaminophen. He had a past history of depression and colitis, and had no known drug allergies. Magnetic resonance imaging had revealed prior posterior decompression of posterior fusion at the L5/S1 level with metallic hardware artifacts with grade 1 anterolisthesis with posterior uncovering of the disc and superimposed left paracentral protrusion. There was also enhancing tissue encasing the thecal sac and the S1 nerve roots at the L5-S1 level.

The patient had no sustained improvement with transforaminal epidural injections. Following a one-week trial of SCS, he reported excellent relief and was subsequently implanted with dual eight-contact leads and the implantable EON ${ }^{\mathrm{TM}}$ pulse generator was placed in the left buttock region. After two weeks, the patient complained of increased pain from the mid back incision. On physical examination, there was trace serosanguinous drainage, without significant erythema or pus. He was afebrile but had a white blood cell count of 14.6. The differential revealed $83.9 \%$ neutrophils and $12.4 \%$ lymphocytes, with no bands. The patient was treated for a presumptive superficial wound infection with antibiotics for ten days with resolution of the pain and white count.

However, despite resolution of the infection, the patient still had a skin inflammatory change that persisted for 5 weeks following implantation (Figure 4). Because of poor

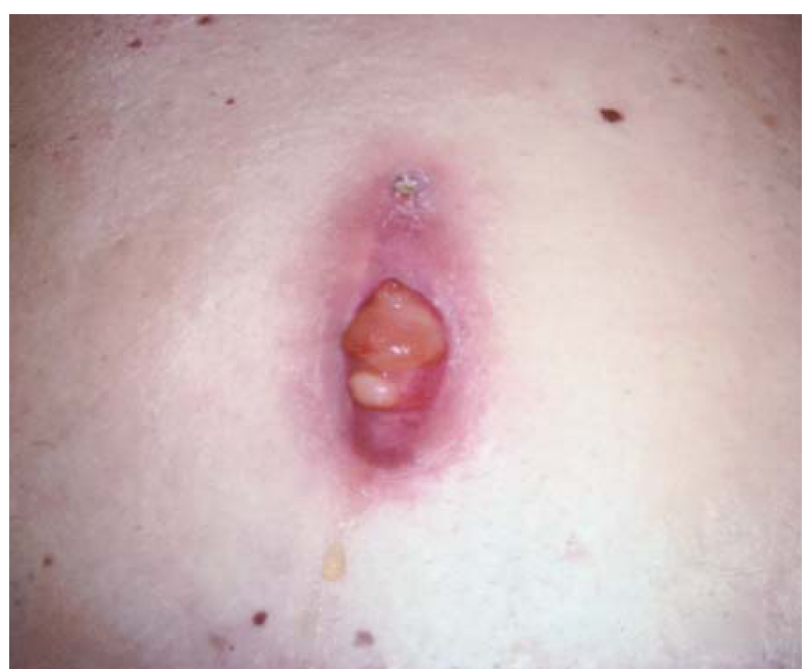

Figure 4 Persistent inflammatory reaction on the upper back (L2 level) 5 weeks post implantation in case 2 . Lead strain relief is directly under the inflammatory tissue. 
wound healing, the decision was made to remove the dual leads, silastic anchors with associated 0 silk sutures, and the implantable pulse generator. Intraoperative frozen sections revealed acute and chronic inflammation, with a foreign body giant cell reaction (Figure $5 \mathrm{~A}$ and $\mathrm{B}$ ). The removal of the soft inflammatory tissue in Figure 4 revealed the underlying leads, and the pocket floor of the implantable pulse generator that had excess lead coils was notable for soft tissue inflammation. Similarly, the tissue was very friable and bled easily with manipulation. Interestingly, there was no fibrotic tissue deposition surrounding the leads, anchors, or the floor of the implantable pulse generator pocket. Normal wound healing then proceeded with an uneventful recovery.

\section{Case 3}

The patient was a 40-year-old right-handed female, who presented to the pain center with a diagnosis of complex
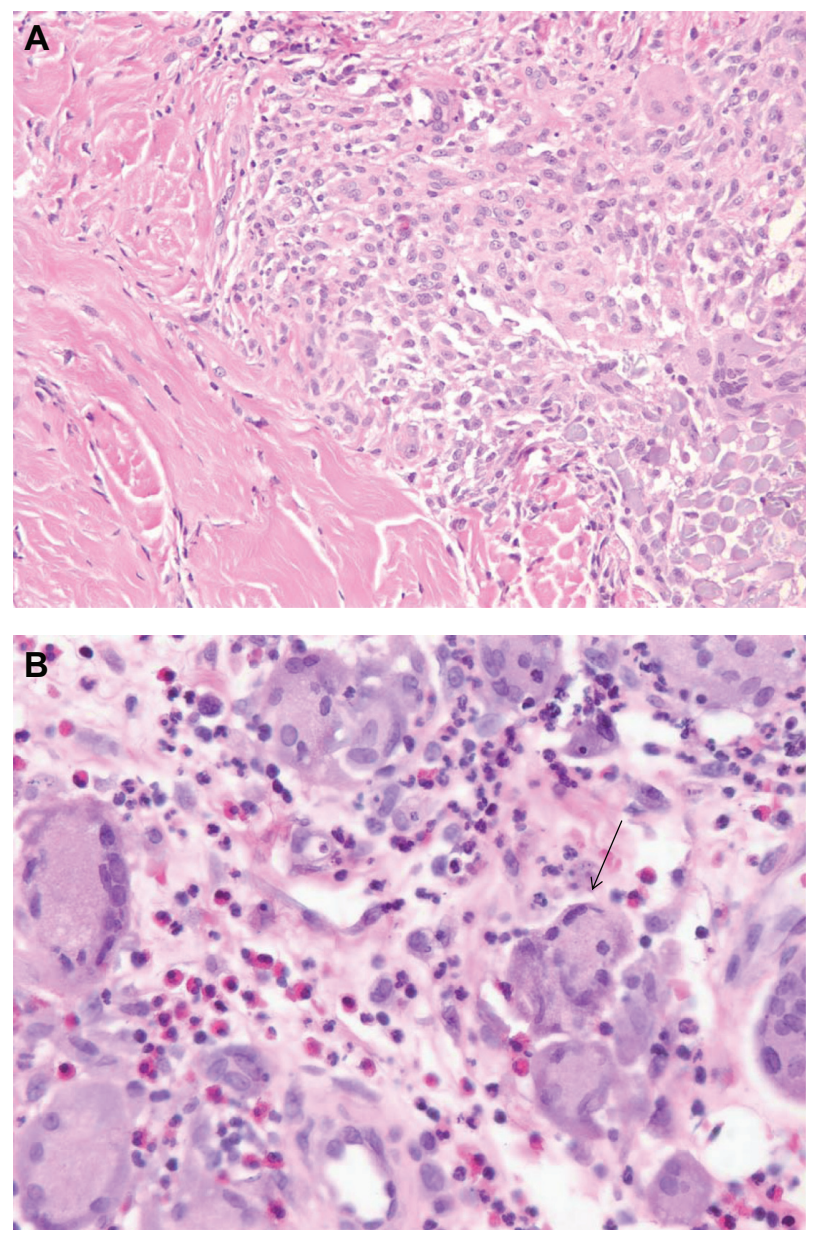

Figure 5 (A) Histopathologic examination of skin and soft tissue specimen taken from the mid back (Figure 4), showing skin and subcutaneous tissue with ulceration and necrosis, acute and chronic inflammation, and a foreign body multinucleated giant cell reaction. (B) Arrow points to one of the many multinucleated giant cells seen close up from tissue detailed in (A). regional pain syndrome affecting the right upper extremity. This condition was believed to be the result of two right shoulder operations for supraspinatus tendon tears. The pain was burning diffusely across her right shoulder, extending over her scapula and down her arm into her hand, with an intensity of $7 / 10$. Her past history was significant only for asthma and sinus infections. She was allergic to nickel, oxycodone/acetaminophen, metronidazole, scopolamine, and tramadol. After multiple interventions including stellate and interscalene blocks, transcutaneous electrical nerve stimulation, physical therapy, intravenous lidocaine, and multimodal pharmacotherapy failed to bring appreciable relief, she underwent a one-week trial of SCS. After reporting a greater than $50 \%$ pain intensity reduction, she had a fully implanted dual 8 contact system placed via an upper back incision at the T2/3 level, with the implantable EON pulse generator placed in the right buttock region. A small pocket for lead extensions was made on the right flank just below the bra line at the T9 level.

The patient was seen one week after implantation and all wounds were healing well. She had reported some clear drainage from the upper back wound, but there was none noted on examination. Her white blood cell count was within normal range and she was afebrile. Five weeks later she called to say that she was developing a partially opened wound at the lead insertion site as well as the lead extension site, with copious amounts of clear drainage. She remained afebrile without chills, and denied a history of trauma or falls. Examination of the wound revealed minimal erythema with no hardware exposure but it had grossly evident soft tissue inflammation. Her white blood cell count was 8.7 but she had an erythrocyte sedimentation rate of 53 and a C-reactive protein of 11.8. Because of persistent wound inflammation and intermittent clear drainage, the leads, silastic anchors (associated 0 Ethibond sutures, Ethicon Inc), and lead extensions were removed 7 weeks post implantation. The clinical plan was to reinsert the leads at a lower level if it was proven to be less immunoreactive using the methods described in case 1. Figure 6A and B displays the histology of the specimen results sent from the upper back wound. The wounds healed rapidly with removal of the leads and anchors.

One month after removal of the leads, the patient called to report a minor amount of bleeding from the buttock wound where the implantable pulse generator had been implanted. She reported no falls or trauma. On examination, the buttock wound had progressed to early dehiscence with clear drainage. She was afebrile and her white blood cell count was 8.1, erythrocyte sedimentation rate was 45 , and C-reactive protein 

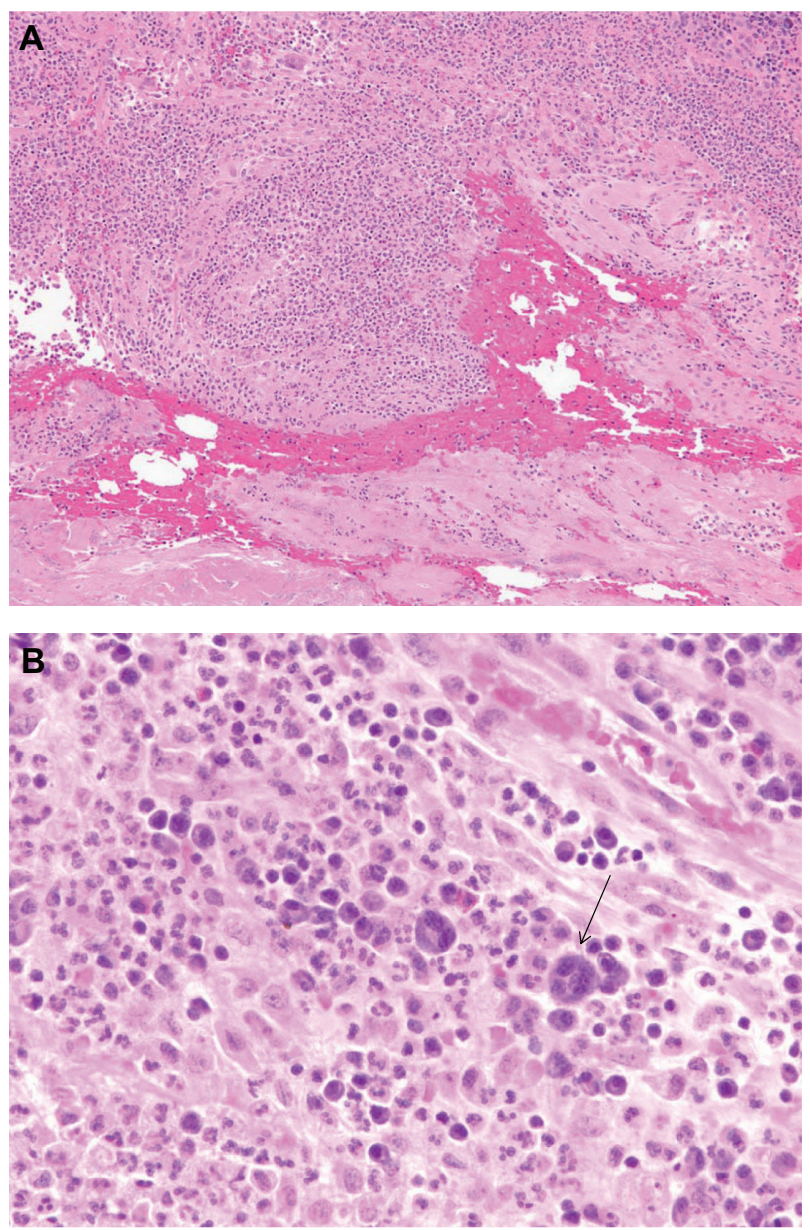

Figure 6 (A) Histopathology results for a specimen taken from the T2/3 level of the back (lead insertion wound). Microscopic analysis reveals granulation tissue with hemorrhage, acute and chronic inflammation, and a focal foreign body giant cell reaction. (B) Arrow points to multinucleated giant cell seen close up from tissue detailed in (A)

was 13.8. The implantable pulse generator was subsequently removed at approximately 11 weeks post implantation. An inflammatory reaction of the pocket floor was noted with very friable tissue (Figure 7). The fibrous floor of the pocket that is usually well formed at 3 months post-implantation was disrupted by inflammation. There were no signs of purulence or infection. A week later, all her wounds were healing well and the drainage had ceased from the buttock.

\section{Discussion}

Allergic or other inflammatory reactions to the components of spinal cord stimulation systems have only rarely been reported. ${ }^{6-8} \mathrm{~A}$ search of the literature revealed only one recent detailed case series to our knowledge, ie, a report from France describing two cases of cutaneous eruption related to spinal cord stimulators. ${ }^{6}$ The first of these cases was a foreign body type reaction to the silicone component

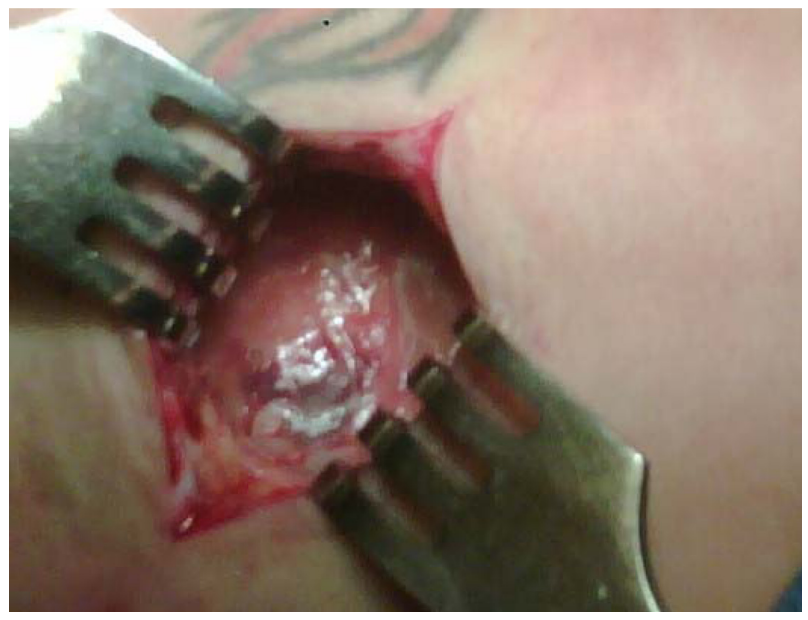

Figure 7 Open wound of the right buttock with implantable pulse generator. The floor of the pocket has modest fibrous tissue with a collection of friable reactive tissue.

of the neurostimulatory electrodes, with histology of the affected tissue showing a foreign body granuloma formation. A similar foreign body granuloma reaction occurred after device removal at the surgical scar, in response to silicone particles. The second case was that of a contact dermatitis reaction thought to be due to the silicone part of the stimulator, with histology of affected tissue showing a contact dermatitis pattern. Delayed hypersensitivity patch testing confirmed a specific sensitivity to silicone.

While reports of inflammatory reactions to spinal cord stimulator devices are exceedingly rare, reactions to other devices, such as pacemakers and cardiac defibrillators, have been reported less rarely. ${ }^{9}$ The apparent higher rate of inflammatory reaction to cardiac rhythm management devices compared with SCS may in part relate to the fact that the former has a higher implantation rate. Two types of delayed inflammatory responses might occur in response to implanted devices, ie, delayed hypersensitivity responses to a specific antigen (eg, the metal polyurethane or silicone rubber of a device) or foreign body giant cell granuloma reactions to device material(s). Delayed hypersensitivity responses to an allergen are mediated by $\mathrm{T}$ cells and monocytes/macrophages, rather than antibodies. Contact dermatitis is a form of delayed hypersensitivity reaction to antigen at the surface of the skin, but a similar process can occur to substances inoculated intradermally or subdermally. The patient in case 1 developed such a reaction likely to the polyurethane lead extensions because the rash was along the entire length of their course. This rash was treated successfully with topical hydrocortisone, but the patient presented within 2 weeks with wound dehiscence 
predisposing to early infection as suggested by the cultures and inflammatory histology. We suspect that the proximity of hydrocortisone treatment to the healing wounds coupled with an inflammatory reaction likely reduced the tensile strength, leading to dehiscence. While contact dermatitis presented approximately one month after implantation, it may present as soon as 8 days, as recently reported after implantation of a peripheral nerve stimulator. ${ }^{10}$

For contact dermatitis (delayed hypersensitivity reactions to items on the surface of the skin), patch testing is a diagnostic technique that can be utilized. This involves placing a suspected substance on the surface of the skin for 48 hours, then looking for an inflammatory response of the skin at 48-96 hours. While this technique can be helpful in some cases, even with contact dermatitis, there can be false negatives and false positives. There is little known about whether patch testing can predict responses to a device that is implanted subcutaneously. The immune system response that occurs with absorption of an allergen from the surface of the skin through the epidermis can be different from that which occurs when the same substance is implanted. The literature provides little insight on the reliability of patch testing to predict an inflammatory response to implanted devices. Because of the authors' prior experience of false negatives on skin patch testing and the aforementioned complications of case 1 , it was thought necessary to try an "in vivo" test of a piece of lead extension in another site for placement of the implantable pulse generator (Figure 3). Interestingly, this 2-month test of implantation of the lead extension fragment in the buttock region did not result in any reaction, and the device was successfully implanted. We are unable to explain why an inflammatory dermatitis occurred in the flank/abdominal region but was absent in the back and buttock area.

In addition to contact dermatitis, a delayed hypersensitivity reaction may manifest as a granuloma, as reported in the French case series. With delayed hypersensitivity reactions, an antigen is taken up by macrophages or monocytes and is then presented to $T$ cells which can specifically recognize that antigen (ie, there is memory from previous exposure to the antigen). This leads to recruitment of further inflammatory cells to the area, including macrophages that can in turn form giant cells. Overall, this inflammatory pattern involving $\mathrm{T}$ cells and macrophages is referred to as a granuloma.

A foreign body reaction can invoke a similar inflammatory pattern, but it is not in response to a particular immunologically recognized antigen. The inflammatory reaction in response to a foreign body starts as the body tries to respond to the foreign substance by attempting to clean the substance out from the body. Neutrophils and macrophages attempt to phagocytose the foreign substance. Macrophages will remain at the foreign body site for an extended period of time, and recruit other inflammatory cells to the area via secretion of chemokines. Granulation tissue can form at the site of the foreign body reaction (similar to granulation tissue seen with wound healing, but in a foreign body reaction, the healing process is unable to complete itself). Therefore, the foreign body reaction consists of persistent inflammation, characterized by foreign body giant cells and granulomas seen on histology. Foreign body giant cells form when macrophages encounter a large foreign body (such as the components of an SCS implant). Because the macrophage cells cannot phagocytose the foreign body, the cells fuse together to form a "giant cell" composed of many fused macrophages. In this case series, the histology of the affected tissue from cases 2 and 3 was that of a foreign body giant cell reaction (Figure $5 \mathrm{~A}$ and $\mathrm{B}$, and Figure $6 \mathrm{~A}$ and $\mathrm{B}$ ). In case 2, the clinical impression is that the polyurethane leads are the likely allergens, given that the inflammatory response was seen in the implantable pulse generator pocket floor where the excess lead coils are placed. However, the silicone anchors cannot be excluded. Regarding case 3 , the polyurethane-coated leads and extensions are even more likely to be the offending stimulus, given that the reaction was in all three wounds, including lead insertion, lead extension, and implantable pulse generator. Component materials, which include silicone rubber (eg, anchors), polyurethane (leads, extensions), titanium (implantable pulse generator), and platinum/iridium (electrodes), are part of an "allergy test kit" (St Jude Medical Neuromodulation) and can be applied topically as a skin patch test. ${ }^{11}$ Among the current three vendors, materials used for the components of SCS devices are similar on account of regulation by the US Food and Drug Administration. No further skin patch or "in vivo" testing was pursued because, given the location of the inflammatory reaction, it was not felt that allergy testing would alter future clinical decision-making in cases 2 and 3. All patients who receive an implanted device develop some degree of foreign body reaction around the device. Why some patients but not others develop a substantial, pathologic, and clinically detrimental level of reaction is not fully known. ${ }^{12,13}$ Interestingly, none of the three patients in this case series had a history of prior allergies to metal, rubber, or autonomic dysfunction to suggest predisposition to cutaneous reactions.

\section{Conclusion}

In summary, delayed inflammatory responses to the components of SCS devices can manifest via T cell/ 
monocyte-mediated delayed hypersensitivity reactions or foreign body giant cell reactions. Contact dermatitis, granuloma formation, and foreign body reactions with giant cell formation are possible in response to SCS devices. The role of skin patch testing remains uncertain when testing for foreign body reactions because these occurred one month after implantation. While the lead/extension polyurethane component is suspected as the most immunogenic source in this case series, other materials of the SCS device cannot be excluded. Excision of the inflamed tissue and histologic evaluation is the key for diagnosis and distinguishing between infection and inflammation. Infection may occur as a complication of poor wound healing because of an underlying inflammatory response to the component(s) of the SCS device.

\section{Disclosure}

The authors report no conflicts of interest in this work.

\section{References}

1. North RB, Kidd DH, Farrokhi F, Plantadosi SA. Spinal cord stimulation versus repeated lumbosacral spine surgery for chronic pain: a randomized controlled trial. Neurosurgery. 2005;56:98-107.

2. Kumar K, Hunter G, Demeria D. Spinal cord stimulation in treatment of chronic benign pain: Challenges in treatment planning and present status, a 22-year experience. Neurosurgery. 2006;58:481-496.

3. Turner JA, Loeser JD, Deyo RA, Sanders SB. Spinal cord stimulation for patients with failed back surgery syndrome or complex regional pain syndrome: a systematic review of effectiveness and complications. Pain. 2004;108:137-147.
4. Kumar K, Taylor RS, Jacques I, et al. Spinal cord stimulation versus conventional medical management for neuropathic pain: a multicentre randomized controlled trial in patients with failed back surgery syndrome. Pain. 2007;132:179-188.

5. Cameron T. Safety and efficacy of spinal cord stimulation for the treatment of chronic pain: a 20 -year literature review. J Neurosurg. 2004;100(Suppl Spine 3):254-267.

6. Delaplace M, Maitre F, Dufour T, et al. Two cases of cutaneous erythema following spinal cord stimulation. Ann Dermatol Venereol. 2010;137:297-300.

7. Ochani TD, Almirante J, Siddiqui A, Kaplan R. Allergic reaction to spinal cord stimulator. Clin J Pain. 2000;16:178-180.

8. McKenna KE, McClean G. Dermatitis induced by a spinal cord stimulator implant. Contact Dermatitis. 1999;41:229.

9. Honari G, Ellis SG, Wilkoff BL, Aronica MA, Svennson LG, Taylor JS Hypersensitivity reactions associated with endovascular devices. Contact Dermatitis. 2008;59:7-22.

10. Zhou L, Lee A, Schaffner R, Dworkin G. Allergic reaction to a peripheral nerve stimulator. Open Neurosurg J. 2012;5:12-15.

11. St Jude Medical. Eon Mini Neuromodulation System Clinician's Manual. Allergy Test Kit, Part Number 64-5953-01. Plano, TX: St Jude Medical; 2010.

12. Greenhalgh DG. The role of monocytes/macrophages in wound healing. In: Robinson JP, Babcock GF, editors. Phagocyte Function: A Guide for Research and Clinical Evaluation. New York, NY: Wiley-Liss Inc; 1998.

13. Anderson JM, Gristina AG, Hanson SR, et al. Host reactions to biomaterials and their evaluation. In: Ratner BD, Hoffman AS, Schoen FJ, Lemons JE, editors. Biomaterials Science: An Introduction to Materials in Medicine. San Diego, CA: Academic Press; 1996.
Journal of Pain Research

\section{Publish your work in this journal}

The Journal of Pain Research is an international, peer-reviewed, open access, online journal that welcomes laboratory and clinical findings in the fields of pain research and the prevention and management of pain. Original research, reviews, symposium reports, hypothesis formation and commentaries are all considered for publication.

\section{Dovepress}

The manuscript management system is completely online and includes a very quick and fair peer-review system, which is all easy to use. Visit http://www.dovepress.com/testimonials.php to read real quotes from published authors. 